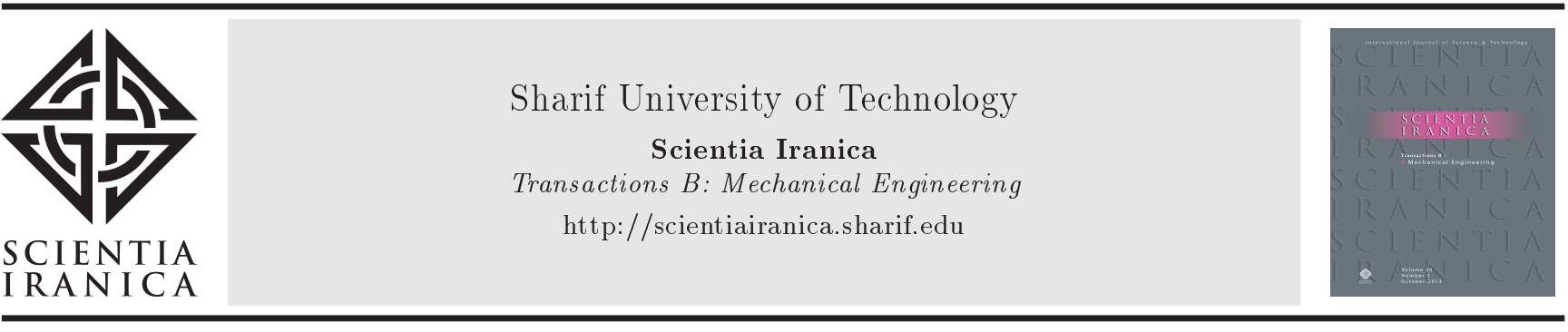

\title{
Size dependent analysis of tapered FG micro-bridge based on a 3D beam theory
}

\author{
Sh. Haddad, M. Baghani*, and M.R. Zakerzadeh \\ School of Mechanical Engineering, College of Engineering, University of Tehran, Tehran, Iran.
}

Received 6 October 2018; received in revised form 9 July 2019; accepted 14 September 2019

\section{KEYWORDS}

Micro-bridge;

3D beam theory;

Modified couple

stress;

Tapered beam;

Analytical HAM

solution.

\begin{abstract}
In the current study, an analytical solution based on a modified couple stress theory for a nonlinear model describing the couple 3D motion of a functionally graded tapered micro-bridge is presented. The small scale effects and the nonlinearity arising from the mid-plane stretching are taken into consideration. Governing equations of motion are derived utilizing modified couple stress theory and applying the Hamilton principle. Dynamic and static analyses to determine the effects of lateral distributed forces and mid-plane stretching are investigated. Towards this aim, analytical the Homotopy-pade technique is employed to capture the nonlinear natural frequencies in high amplitude vibrations of tapered micro-bridges with different types of geometry and material composition. The obtained results of frequencies propose that there is good agreement between the present analytical results and the numerical ones, as opposed to the well-known multiple-scale method. Furthermore, comparing the results in 2D and 3D analyses shows that in $2 \mathrm{D}$ analysis, the stiffness and natural frequency of the micro-beam is underestimated and it is observed that increasing the tapered ratio has different impacts on natural frequencies for micro-beams with different slender ratios.
\end{abstract}

(C) 2020 Sharif University of Technology. All rights reserved.

\section{Introduction}

Micro-beams are of major importance in the fields of micro/nano electro-mechanical systems such as those in sensors and actuators [1]. Beams used in these applications have thicknesses in the order of microns and sub-microns. The size dependent behavior of these structures has been empirically observed by several researchers [2-5]. Incorporating the concept of the length scale parameter, an experimentally measurable

\footnotetext{
*. Corresponding author. Tel.: +982161119921 E-mail addresses: s.haddad@ut.ac.ir (Sh. Haddad); baghani@ut.ac.ir (M.Baghani); zakerzadeh@ut.ac.ir (M.R. Zakerzadeh)
}

property accounting for dislocation and distortion of constitutive crystals and the grain size of a specific material [6] has led to many investigations on size effect phenomena over the past few decades, and some non-classical continuum theories have been developed. Mindlin [7] introduced the higher-order gradient theory, regarding the first and second derivatives of the strain tensor, for elastic materials. Subsequently, using Mindlin's formulation, the strain gradient theory was proposed by Fleck and Hutchinson [8-10] ,which considers only the first derivatives of the strain tensor. Ansari [11] developed a model using the modified strain gradient theory to describe the linear and nonlinear vibrational behavior of fractional viscoelastic Timoshenko micro/nano beams. In this paper, a predictorcorrector technique is utilized to deal with the set of nonlinear governing equations. Based on strain 
gradient elasticity theory, an analytical solution is proposed for an Euler-Bernoulli FG nano beam by Li et al. [12]. In this study, the device is lying on an elastic foundation and the bending and buckling behavior are investigated.

Another well-known theory which involves size effects, is the couple stress theory introduced by Mindlin and Tiersten [13].

The modified couple stress theory proposed by Yang et al. [6] considers the equilibrium equation of forces and couples, and moments of couples, applied to a single material element. In this theory, two classical and one additional material constant are introduced to reflect the microstructure-related size influences.

Based on the modified couple stress theory, the dynamic and static response of homogeneous and Functionally Graded Materials (FGMs), including linear vibration, elastic bending, post buckling and nonlinear vibration, have been investigated by many researchers [14-17]. FGMs are inhomogeneous composites of two different constituents, typically metal and ceramic, with a desired continuous change in compositional characteristics as a function of position along specified dimensions. Application of FGMs has been widely extended in various industrial fields, especially in designing micro and nano systems such as thin films in the form of shape memory alloys [18,19] Atomic Force Microscopes (AFMs) [20], and micro/nano electro-mechanical systems [21-25].

In addition to FG functionality in micro beams, non-prismatic beams, i.e. beams with a varying crosssection (abruptly or gradually) along the length of the beam, play an important role in different fields [26], in which they can be used architecturally and aesthetically in aspects of engineering design to optimize the strength and weight of the structure. One of the effective applications of tapered micro beams is in the micro energy harvester, in which tailoring the structural parameters of the cantilever beam could lead to an increased harvester power [27] and can considerably influence the natural frequency of microstructures [28]. Baghani et al. [26] by considering the assumption of the inextensibility of the neutral axis, studied large amplitude free vibrations of tapered micro-bridges on a nonlinear elastic foundation. Dynamic analysis of a parabolic tapered Euler-Bernoulli cantilever beam under a travelling mass is accomplished by Zhao et al. [29].

In most investigations performed on the behavior of micro and sub micro beams, the problem is considered a $2 \mathrm{D}$ case, so there is a geometric imperfection, since the beam is under 3D deformation and themid plane stretching may lead to a coupling between the displacements of lateral directions. Due to the importance of micro-beams and micro-systems, the couple 3D deformations of these structures have been studied by many researchers. Considering mid plane stretching and geometric non-linearity, Mojahedi et al. [30,31], and Mojahedi and Rahaeifard [32] studied 3 dimensional motion for the micro/nano beam and reported the static, dynamic and instability behavior of an electro-static actuated bridge and cantilever micro/nano gyroscope and micro bridges.

The Euler Bernoulli beam theory is the most wellknown for calculating beam deformation with a slender ratio, commonly, $L / h>10$. According to this theory, due to ignoring shear strains, a plane after deformation remains a plane and still perpendicular to the central line of the beam. Employing the modified couple stress theory in the Euler-Bernoulli beam model, the results show larger bending stiffness than in classical ones [33]. Euler-Bernoulli beam theory can predict the lateral and bending deformation. However, in this paper, a strain tensor is built to include the deformation in both lateral and in axial directions.

The aim of is this paper, firstly, is to present a mechanical model to illustrate the $3 \mathrm{D}$ motion of an FGM double tapered micro-bridge. Subsequently, the impacts of nonlinearity caused by considering the third dimension in the calculations, and effects of material composition, tapered ratio and geometrical properties on the static and dynamic responses are investigated. An analytical Homotopy-pade method is implemented to estimate the natural frequencies of the micro-bridges. As will be discussed in the paper, the Homotopy method is more general than multiple scale methods employed in many nonlinear problems [34]. In the Multiple Time Scale (MTS) technique, using a small physical parameter, the nonlinear problems are transferred into a sequence of linear perturbed problems. This small parameter is not needed in Homotopy Analysis Method (HAM) and gaining higher order approximations are easier, leading to smaller computational cost. In addition to low simulation cost, here, we demonstrate that HAM is more appropriate for strong nonlinearities and high amplitudes. For this purpose, a comparison study is undertaken to capture the accuracy of HAM as opposed to MTS, as a classic perturbation method. The consistency of calculated analytical results with numerical ones is also presented.

\section{Problem formulation}

Figure 1 shows two views of a FG tapered micro-bridge under lateral loads. The deformation and distributed load in the $z$ direction are depicted by $\hat{w}(\hat{x}, \hat{t}), \hat{f}_{z}$, and in a $y$ direction by $\hat{v}(\hat{x}, \hat{t})$ and $\hat{f}_{y}$, respectively. The strain energy density, employing the couple stress theory, is written as:

$$
\tilde{v}=\frac{1}{2}\left(\sigma_{i j} \varepsilon_{i j}+\chi_{i j} m_{i j}\right),
$$




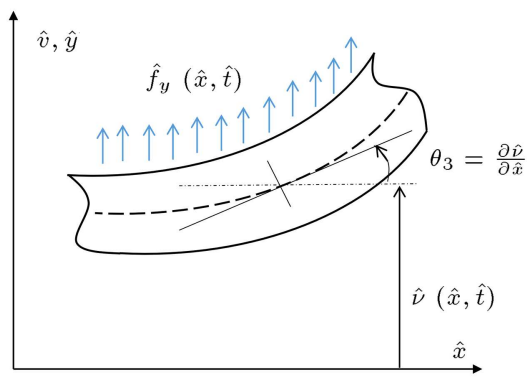

(a)

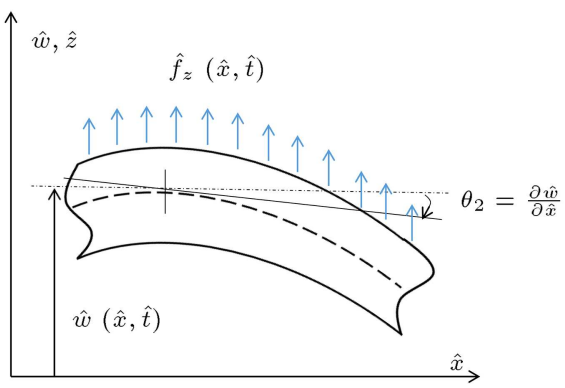

(b)

Figure 1. A schematic view of micro-bridge under distributed forces in lateral directions.

where $\sigma_{i j}$ and $\varepsilon_{i j}$ are the components of stress and strain tensor, respectively, $m_{i j}$ is the deviatoric part of the couple stress tensor and $\chi_{i j}$ is the symmetric curvature tensor.

Assuming flexible motions in both directions, the relation between transverse displacements and the rotation vector components becomes:

$$
\vec{\theta}=-\frac{\partial \hat{w}}{\partial \hat{x}} \hat{e}_{2}-\frac{\partial \hat{v}}{\partial \hat{x}} \hat{e}_{3} .
$$

The non-zero components of the deviatoric part of the couple stress tensor and the symmetric curvature tensor are recorded as:

$$
\left\{\begin{array} { l } 
{ \chi _ { i j } = \frac { 1 } { 2 } ( \theta _ { i , j } + \theta _ { j , i } ) } \\
{ m _ { i j } = 2 l ^ { 2 } \mu \chi _ { i j } }
\end{array} \rightarrow \left\{\begin{array}{l}
m_{x y}=m_{y x}=-\mu l^{2} \frac{\partial^{2} \hat{w}}{\partial \hat{x}^{2}} \\
m_{x z}=m_{z x}=-\mu l^{2} \frac{\partial^{2} \hat{\hat{x}}}{\partial \hat{x}^{2}}
\end{array}\right.\right.
$$

In which $l$ and $\mu$ symbolize the material length scale parameter and the shear modulus, respectively. The displacement field according to the Euler-Bernoulli beam theory for a micro-beam subjected to a distributed load in both lateral directions can be written as [35]:

$$
u_{1}=\hat{u}-\hat{z} \frac{\partial \hat{w}}{\partial \hat{x}}-\hat{y} \frac{\partial \hat{v}}{\partial \hat{x}}, \quad u_{2}=\hat{v}(\hat{x}), \quad u_{3}=\hat{w}(\hat{x}),
$$

where $\hat{u}$ is the axial deformation. Utilizing the vonKarman relation, the nonzero strain (i.e. the axial strain) is derived [32]:

$$
\varepsilon_{x}=\frac{\partial \hat{u}}{\partial \hat{x}}-\hat{z} \frac{\partial^{2} \hat{w}}{\partial \hat{x}^{2}}-\hat{y} \frac{\partial^{2} \hat{v}}{\partial \hat{x}^{2}}+\frac{1}{2}\left(\frac{\partial \hat{v}}{\partial \hat{x}}\right)^{2}+\frac{1}{2}\left(\frac{\partial \hat{w}}{\partial \hat{x}}\right)^{2} .
$$

Thus, the axial stress in the beam would be:

$$
\begin{aligned}
\sigma_{x}= & E\left[\frac{\partial \hat{u}}{\partial \hat{x}}-\hat{z} \frac{\partial^{2} \hat{w}}{\partial \hat{x}^{2}}-\hat{y} \frac{\partial^{2} \hat{v}}{\partial \hat{x}^{2}}+\frac{1}{2}\left(\frac{\partial \hat{v}}{\partial \hat{x}}\right)^{2}\right. \\
& \left.+\frac{1}{2}\left(\frac{\partial \hat{w}}{\partial \hat{x}}\right)^{2}\right],
\end{aligned}
$$

in which $E$ is the young modulus of elasticity.

The potential and kinetic energy of the beam according to Eq. (1) is written, respectively, as:

$$
\begin{gathered}
V=\int_{0}^{L}\left[\iint_{A} \tilde{v} d A\right] d \hat{x}=\frac{1}{2} \int_{0}^{L}\left[\int \int _ { A } \left(\sigma_{x} \varepsilon_{x}+\chi_{x y} m_{x y}\right.\right. \\
\left.\left.+\chi_{y x} m_{y x}+\chi_{x z} m_{x z}+\chi_{z x} m_{z x}\right) d A\right] d \hat{x}, \quad(7) \\
T=\frac{1}{2} \int_{0}^{L} \rho(\hat{x}) A(\hat{x})\left[\left(\frac{\partial \hat{u}}{\partial \hat{t}}\right)^{2}+\left(\frac{\partial \hat{v}}{\partial \hat{t}}\right)^{2}+\left(\frac{\partial \hat{w}}{\partial \hat{t}}\right)^{2}\right] d \hat{x},
\end{gathered}
$$

where $\rho(\hat{x}), A(\hat{x})$, and $L$ are the mass per unit volume, cross section and the length of the beam, respectively.

Considering the work of external forces on the micro bridge as $w_{\text {ext }}=\int_{0}^{L}\left[\hat{f}_{y} \hat{v}+\hat{f}_{z} \hat{w}\right] d \hat{x}$, the Lagrangian of the motion, then, is given as:

$$
\begin{aligned}
L= & T-V+W_{e x t}=\frac{1}{2} \int_{0}^{L} \rho(\hat{x}) A(\hat{x}) \\
& {\left[\left(\frac{\partial \hat{u}}{\partial \hat{t}}\right)^{2}+\left(\frac{\partial \hat{v}}{\partial \hat{t}}\right)^{2}+\left(\frac{\partial \hat{w}}{\partial \hat{t}}\right)^{2}\right] d \hat{x}-\int_{A} E(\hat{x}) } \\
& {\left[\begin{array}{c}
\left(\frac{\partial \hat{u}}{\partial \hat{x}}-\hat{z} \frac{\partial^{2} \hat{w}}{\partial \hat{x}^{2}}-y \frac{\partial^{2} \hat{v}}{\partial \hat{x}^{2}}+\frac{1}{2}\left(\frac{\partial \hat{v}}{\partial \hat{x}}\right)^{2}+\frac{1}{2}\left(\frac{\partial \hat{w}}{\partial \hat{x}}\right)^{2}\right)^{2} \\
-\mu(\hat{x}) l(\hat{x})^{2}\left(\frac{\partial^{2} \hat{v}}{\partial \hat{x}^{2}}\right)^{2}-\mu(\hat{x}) l(\hat{x})^{2}\left(\frac{\partial^{2} \hat{w}}{\partial \hat{x}^{2}}\right)^{2}
\end{array}\right] } \\
& d \hat{x}+\int_{0}^{L}\left[\hat{f}_{y} \hat{v}+\hat{f}_{z} \hat{w}\right] d \hat{x} .
\end{aligned}
$$

Applying the Hamilton principle, one has :

$$
\delta \int_{0}^{\hat{t}} L d \hat{t}=0
$$

Expanding the integral in Eq. (10), the governing equations of motion for the doubly clamped microbeam are as follows:

$$
\begin{aligned}
& \frac{\partial}{\partial \hat{x}}\left(E(\hat{x}) A(\hat{x})\left(\frac{\partial \hat{u}}{\partial \hat{x}}+\frac{1}{2}\left(\frac{\partial \hat{v}}{\partial \hat{x}}\right)^{2}+\frac{1}{2}\left(\frac{\partial \hat{w}}{\partial \hat{x}}\right)^{2}\right)\right) \\
& =\rho(\hat{x}) A(\hat{x}) \frac{\partial^{2} \hat{u}}{\partial \hat{t}^{2}}
\end{aligned}
$$




$$
\begin{aligned}
& \rho(\hat{x}) A(\hat{x}) \frac{\partial^{2} \hat{v}}{\partial \hat{t}^{2}}+\frac{\partial^{2}}{\partial \hat{x}^{2}}\left(\left(\mu(\hat{x}) A(\hat{x}) l(\hat{x})^{2}\right.\right. \\
& \left.\left.+E(\hat{x}) I_{z}(\hat{x})\right) \frac{\partial^{2} \hat{v}}{\partial \hat{x}^{2}}\right)-\frac{\partial}{\partial \hat{x}}\left(E ( \hat { x } ) A ( \hat { x } ) \left(\frac{\partial \hat{u}}{\partial \hat{x}}+\frac{1}{2}\right.\right. \\
& \left.\left.\left(\frac{\partial \hat{v}}{\partial \hat{x}}\right)^{2}+\frac{1}{2}\left(\frac{\partial \hat{w}}{\partial \hat{x}}\right)^{2}\right) \frac{\partial \hat{v}}{\partial \hat{x}}\right)=\hat{f}_{y}, \\
& \rho(\hat{x}) A(\hat{x}) \frac{\partial^{2} \hat{w}}{\partial \hat{t}^{2}}+\frac{\partial^{2}}{\partial \hat{x}^{2}}\left(\left(\mu(\hat{x}) A(\hat{x}) l(\hat{x})^{2}\right.\right. \\
& \left.\left.+E(\hat{x}) I_{y}(\hat{x})\right) \frac{\partial^{2} \hat{w}}{\partial \hat{x}^{2}}\right)-\frac{\partial}{\partial \hat{x}}\left(E ( \hat { x } ) A ( \hat { x } ) \left(\frac{\partial \hat{u}}{\partial \hat{x}}\right.\right. \\
& \left.\left.+\frac{1}{2}\left(\frac{\partial \hat{v}}{\partial \hat{x}}\right)^{2}+\frac{1}{2}\left(\frac{\partial \hat{w}}{\partial \hat{x}}\right)^{2}\right) \frac{\partial \hat{w}}{\partial \hat{x}}\right)=\hat{f}_{z} .
\end{aligned}
$$

In Eq. (14), the appropriate boundary conditions are given for the doubly clamped microbridge:

$$
\begin{aligned}
& \hat{u}(0, \hat{t})=\hat{u}(\hat{L}, \hat{t})=0, \quad \hat{v}(0, \hat{t})=\hat{v}(\hat{L}, \hat{t})=0 \\
& \frac{\partial \hat{v}}{\partial \hat{x}}(0, \hat{t})=\frac{\partial \hat{v}}{\partial \hat{x}}(\hat{L}, \hat{t})=0, \quad \hat{w}(0, \hat{t})=0 \\
& \hat{w}(L, \hat{t})=0, \quad \frac{\partial \hat{w}}{\partial \hat{x}}(0, \hat{t})=0, \quad \frac{\partial \hat{w}}{\partial \hat{x}}(L, \hat{t})=0
\end{aligned}
$$

In the case of no axial forces, longitudinal inertia is negligible [31]. Thus, by solving Eq. (11) and applying the corresponding boundary conditions, one arrives at:

For doubly clamped BCs:

$$
\begin{aligned}
u_{\text {bridge }}(\hat{x}, \hat{t})= & -\int_{0}^{\hat{x}}\left[\frac{1}{2}\left(\frac{\partial \hat{v}}{\partial \hat{x}}\right)^{2}+\frac{1}{2}\left(\frac{\partial \hat{w}}{\partial \hat{x}}\right)^{2}\right] d \hat{x} \\
& +\frac{\int_{0}^{\hat{x}} \frac{1}{E(\hat{x}) A(\hat{x})} d \hat{x}}{\int_{0}^{L} \frac{1}{E(\hat{x}) A(\hat{x})} d \hat{x}} \\
& \int_{0}^{L}\left[\frac{1}{2}\left(\frac{\partial \hat{v}}{\partial \hat{x}}\right)^{2}+\frac{1}{2}\left(\frac{\partial \hat{w}}{\partial \hat{x}}\right)^{2}\right] d \hat{x} .
\end{aligned}
$$

Substituting $u_{\text {bridge }}(\hat{x}, \hat{t})$ from Eq. (15) into Eqs. (12) and (13), the governing equations of motion for a FG tapered micro-beam take the following forms:

$$
\begin{aligned}
& \frac{\partial^{2}}{\partial \hat{x}^{2}}\left(\left(\mu(\hat{x}) A(\hat{x}) l(\hat{x})^{2}+E(\hat{x}) I_{z}(\hat{x})\right) \frac{\partial^{2} \hat{v}}{\partial \hat{x}^{2}}\right) \\
& +\rho(\hat{x}) A(\hat{x}) \frac{\partial^{2} \hat{v}}{\partial \hat{t}^{2}}-\frac{1}{\int_{0}^{L} \frac{1}{E(\hat{x}) A(\hat{x})} d \hat{x}} \\
& {\left[\int_{0}^{L} \frac{1}{2}\left(\frac{\partial \hat{v}}{\partial \hat{x}}\right)^{2}+\frac{1}{2}\left(\frac{\partial \hat{w}}{\partial \hat{x}}\right)^{2} d \hat{x}\right] \frac{\partial^{2} \hat{v}}{\partial \hat{x}^{2}}=\hat{f}_{y}} \\
& \frac{\partial^{2}}{\partial \hat{x}^{2}}\left(\left(\mu(\hat{x}) A(\hat{x}) l(\hat{x})^{2}+E(\hat{x}) I_{y}(\hat{x})\right) \frac{\partial^{2} \hat{w}}{\partial \hat{x}^{2}}\right) \\
& +\rho(\hat{x}) A(\hat{x}) \frac{\partial^{2} \hat{w}}{\partial \hat{t}^{2}}-\frac{1}{\int_{0}^{L} \frac{1}{E(\hat{x}) A(\hat{x})} d \hat{x}} \\
& {\left[\int_{0}^{L} \frac{1}{2}\left(\frac{\partial \hat{v}}{\partial \hat{x}}\right)^{2}+\frac{1}{2}\left(\frac{\partial \hat{w}}{\partial \hat{x}}\right)^{2} d \hat{x}\right] \frac{\partial^{2} \hat{w}}{\partial \hat{x}^{2}}=\underset{(17)}{\hat{f}_{z}}}
\end{aligned}
$$

where $A(\xi)=A_{0} \xi^{2}, I_{y}(\xi)=I_{0_{y}} \xi^{4}, I_{z}(\xi)=I_{0_{z}} \xi^{4}$. As shown in Figure $2, \xi=1+\left(\varepsilon_{0}-1\right) x, \varepsilon_{0}$ is the tapered ratio, and $I_{0 y}$ and $I_{0 z}$ are the moments of inertia about the $y$ and $z$ axes, respectively, at $\xi=1$. $A_{0}$ is also the cross section at $\xi=1$.

Using power-law functions to ensure a smooth distribution of stresses along the longitude direction and all the interfaces, the FG material properties are expected to vary along the axis of the beam as [36]:

$$
\begin{aligned}
& E(\hat{x})=E_{0}+\left(E_{1}-E_{0}\right)(\hat{x} / L)^{n}, \\
& \rho(\hat{x})=\rho_{0}+\left(\rho_{1}-\rho_{0}\right)(\hat{x} / L)^{n}, \\
& v(\hat{x})=v_{0}+\left(v_{1}-v_{0}\right)(\hat{x} / L)^{n}, \\
& l(\hat{x})=l_{0}+\left(l_{1}-l_{0}\right)(\hat{x} / L)^{n}, \\
& \mu(\hat{x})=\frac{E_{0}+\left(E_{1}-E_{0}\right)\left(\hat{x} / L^{n}\right)}{2\left(1+v_{0}+\left(v_{1}-v_{0}\right) \hat{x} / l^{n}\right)},
\end{aligned}
$$

where $n$ is the power index parameter, $v_{0}$ and $v_{1}$ are the Poisson ratios, indices ' 0 ' and ' 1 ' represent the

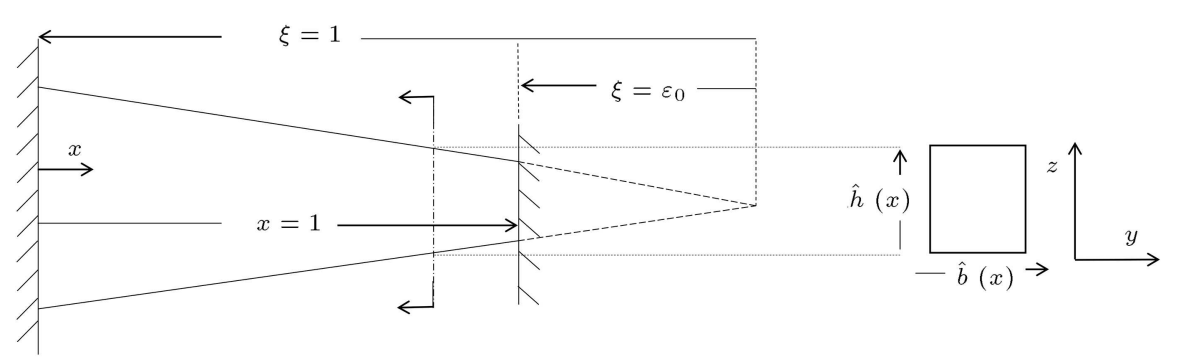

Figure 2. Initial configuration of the tapered micro-bridge. 
material type at $x=0$ and $x=1$ of the micro-beam, respectively.

The following dimensionless parameters are utilized:

$$
\begin{aligned}
& t=\frac{\hat{t}}{\chi}, \quad \chi^{2}=\frac{\rho_{0} A_{0} L^{4}}{E_{0} I_{o y}}, \quad x=\frac{\hat{x}}{L}, \quad w=\frac{\hat{w}}{L}, \quad v=\frac{\hat{v}}{L}, \\
& f_{y}=\frac{\hat{f}_{y} L^{3}}{E_{0} I_{o y}}, \quad f_{z}=\frac{\hat{f}_{z} L^{3}}{E_{0} I_{o y}}, \quad b=\frac{\hat{b}}{L}, \quad h=\frac{\hat{h}}{L}, \quad
\end{aligned}
$$

in which $\hat{h}$ and $\hat{b}$ are the thickness and width of the cross section of the micro-bridge, respectively, as shown in Figure 2. The dimensionless equations of motion are derived as:

$$
\begin{gathered}
\alpha_{y}(x) \omega^{2} \frac{\partial^{2} v}{\partial t^{2}}+\left(\gamma_{y_{2}}(x)-\gamma_{y_{1}}\right) \frac{\partial^{2} v}{\partial x^{2}}+\gamma_{y_{3}}(x) \frac{\partial^{3} v}{\partial x^{3}} \\
+\gamma_{y_{4}}(x) \frac{\partial^{4} v}{\partial x^{4}}=f_{y}, \\
\alpha_{z}(x) \frac{\partial^{2} W}{\partial t^{2}}+\left(\gamma_{z_{2}}(x)-\gamma_{z_{1}}\right) \frac{\partial^{2} W}{\partial x^{2}} \\
+\gamma_{z_{3}}(x) \frac{\partial^{3} W}{\partial x^{3}}+\gamma_{z_{4}}(x) \frac{\partial^{4} W}{\partial x^{4}}=f_{z},
\end{gathered}
$$

where:

$$
\begin{aligned}
& \gamma_{y_{1}}=\frac{L^{2}}{E_{0} I_{0 y}} \frac{\int_{0}^{1} \frac{1}{2}\left(\frac{\partial v}{\partial x}\right)^{2}+\frac{1}{2}\left(\frac{\partial w}{\partial x}\right)^{2} d x}{\int_{0}^{1} \frac{1}{E(x) A(x)} d x} \\
& \gamma_{y_{2}}(x)=\frac{\partial^{2}\left(\mu(x) A(x) l^{2}(x)+E(x) I_{z}(x)\right)}{E_{0} I_{0 y} \partial x^{2}} \\
& \gamma_{y_{3}}(x)=2 \frac{\partial}{E_{0} I_{0 y} \partial x}\left(\mu(x) A(x) l^{2}(x)+E(x) I_{z}(x)\right) \\
& \gamma_{y_{4}}(x)=\frac{\mu(x) A(x) l^{2}(x)}{E_{0} I_{0 y}}+\frac{E(x) I_{z}(x)}{E_{0} I_{0 y}} \\
& \alpha_{y}(x)=\frac{\rho(x) A(x)}{\rho_{0} A_{0}} . \\
& \alpha_{z}(x)=\alpha_{y}(x), \\
& \gamma_{z_{1}}=\gamma_{y_{1}}, \\
& \gamma_{z_{4}}(x)=\frac{\mu(x) A(x) l(x)^{2}}{E_{0} I_{0 y}}+\frac{E(x) I_{y}(x)}{E_{0} I_{0 y}} \\
& \gamma_{z_{2}}(x)=\frac{\partial^{2}\left(\mu(x) A(x) l^{2}(x)+E(x) I_{y}(x)\right)}{E_{0} I_{0 y} \partial x^{2}} \\
& E_{0} I_{0 y} \partial x
\end{aligned}
$$

In this paper, a single pre-assumed mode approach is considered to discretize Eqs. (20) and (21). Assume the deflections $v$ and $w$ are as follows:

$$
\begin{aligned}
& v(x)=q_{y}(t) \phi_{y}(x), \\
& w(x)=q_{z}(t) \phi_{z}(x),
\end{aligned}
$$

where $\phi_{y}(x)$ and $\phi_{z}(x)$ are the normalized, self-similar (i.e. independent of the motion amplitude) assumed mode shapes of the beam in $y$ and $z$ directions, respectively. Accordingly, $q_{y}(t)$ and $q_{z}(t)$ are the generalized coordinates of the assumed deflection modes $\phi_{y}(x)$ and $\phi_{z}(x)$, respectively. Applying the Galerkin decomposition method, the weak forms of Eqs. (20) and (21) are constructed in the forms of:

$$
\begin{aligned}
& \ddot{q}_{y}+\zeta_{y} q_{y}+\beta_{y} q_{y}\left(q_{y}^{2}+\kappa q_{z}^{2}\right)=F_{y}, \\
& \ddot{q}_{z}+\zeta_{z} q_{z}+\beta_{z} q_{z}\left(q_{z}^{2} / \kappa+q_{y}^{2}\right)=F_{z},
\end{aligned}
$$

where:

$$
\begin{aligned}
& \lambda_{y}=\int_{0}^{1} \frac{\rho A}{\rho_{0} A_{0}} \phi_{y}^{2} d x, \quad F_{y}=\frac{1}{\lambda_{y}} \int_{0}^{1} f_{y} \phi_{y} d x \\
& \zeta_{y}=\frac{1}{\lambda_{y}} \int_{0}^{1} \frac{1}{E_{0} I_{0 y}} \frac{\partial^{2}}{\partial x^{2}}\left(\left(\mu A l^{2}+E I_{z}\right) \frac{\partial^{2} \phi_{y}}{\partial x^{2}}\right) \varphi_{y} d x \\
& \lambda_{z}=\int_{0}^{1} \frac{\rho A}{\rho_{0} A_{0}} \phi_{z}^{2} d x, \quad F_{z}=\frac{1}{\lambda_{z}} \int_{0}^{1} f_{z} \phi_{z} d x \\
& \zeta_{z}=\frac{1}{\lambda_{z}} \int_{0}^{1} \frac{1}{E_{0} I_{0 y}} \frac{\partial^{2}}{\partial x^{2}}\left(\left(\mu A l^{2}+E I_{z}\right) \frac{\partial^{2} \phi_{z}}{\partial \hat{x}^{2}}\right)^{\phi_{z} d x} \\
& \beta_{y}=-\left.\frac{E A}{2 \lambda_{y} E_{0} I_{0 y}} \int_{0}^{1} L^{2} \frac{\partial^{2} \phi_{y}}{\partial x^{2}} \phi_{y} d x \cdot\left(\frac{\partial \phi_{y}}{\partial x}\right)^{2}\right|_{x=1} \\
& \kappa=\frac{\left(\frac{\partial \phi_{z}}{\partial x}\right)^{2}}{\left.\left(\frac{\partial \phi_{y}}{\partial x}\right)^{2}\right|_{x=1}}, \\
& \beta_{z}=-\frac{E A}{2 \lambda_{z} E_{0} I_{0 y}} \int_{0}^{1} L^{2} \frac{\partial^{2} \phi_{z}}{\partial x^{2}} \phi_{z} d x .\left.\left(\frac{\partial \phi_{z}}{\partial x}\right)^{2}\right|_{x=1}(29
\end{aligned}
$$

'? represents the derivative with respect to $t$. In this work $\phi_{y}(x)$ and $\phi_{z}(x)$ for a double tapered micro-bridge are defined in the following form $[34,35]$ :

$$
\begin{aligned}
\phi_{z \text { or } y}(\xi)= & \xi^{-1}\left[C_{1} J_{2}(Z)+C_{2} Y_{2}(Z)+C_{3} I_{2}(Z)\right. \\
& \left.+C_{4} K_{2}(Z)\right]
\end{aligned}
$$

where $Z=2 \Lambda \xi^{1 / 2}$, and the values of $\Lambda$ for different tapered ratios are given in Table $1 . J$ and $Y$ are Bessel functions of the first and second kind, respectively, and $I$ and $K$ are modified Bessel functions of the first and second kind, respectively. Arbitrary constants $C_{1}-C_{4}$ are calculated through applying the following boundary conditions in $\hat{y}$ and $\hat{z}$ directions, respectively: 
Table 1. Values of parameter $\Lambda$ for different tapered ratios.

\begin{tabular}{cccccc}
\hline & $\varepsilon_{\mathbf{0}}$ & 0.2 & 0.4 & 0.6 & 0.8 \\
\hline$\Lambda$ & Doubly clamped tapered micro-beam & 4.398 & 6.495 & 10.524 & 22.415 \\
\hline
\end{tabular}

$$
\begin{array}{ll}
\phi_{z \text { or } y}\left(\varepsilon_{0}\right)=0, & \frac{\phi_{z \text { or } y}\left(\varepsilon_{0}\right)}{\partial \xi}=0, \\
\phi_{z \text { or } y}(1)=0, & \frac{\partial \phi_{z \text { or } y}(1)}{\partial \xi}=0 .
\end{array}
$$

It should be noted that by assuming identical mode shapes for both lateral directions, parameter $\kappa$ becomes equal to unity. Eqs. (25) and (26) represent the weak form of the equations, governing the motion of a microbeam, which are modeled based on the proposed assumptions and the modified couple stress theory. In the following sections, analytical and numerical solutions, reflecting the static and dynamic performances of the FG tapered micro-beam, will be investigated.

\section{Static deflection analysis}

In this section, by neglecting the inertia terms from Eqs. (25) and (26), the coupled nonlinear static deflections of the FG tapered micro-beam under lateral distributed force are investigated. The simplified equations for the static analysis are expressed as:

$$
\begin{aligned}
& \zeta_{y} q_{y}+\beta_{y} q_{v}\left(q_{y}^{2}+\kappa q_{z}^{2}\right)=F_{y}, \\
& \zeta_{z} q_{z}+\beta_{z} q_{z}\left(q_{z}^{2} / \kappa+q_{y}^{2}\right)=F_{z} .
\end{aligned}
$$

As can be seen, there are two algebraic coupled nonlinear equations, governing static motions in the transvers directions. A numeric iterative method is utilized to solve simultaneously the above mentioned equations. This iterative procedure is continued until the desired convergence is met.

\section{Free vibration analysis}

In the next section, the approximate analytical solution according to the HAM is employed to arrive at an analytical solution for the non-linear Eqs. (25) and (26), reflecting the dynamic behavior of the represented model. HAM is an approximate analytic technique for solving nonlinear differential equations. In the following section, a brief description of this method is given.

\subsection{Homotopy Analysis Method (HAM)}

To demonstrate the main idea of HAM, consider the general non-linear problem as:

$$
N[q(\tau)]=0,
$$

in which $N$ represents a general non-linear differential operator and $q$ is considered as an unknown function, describing the exact solution to the non-linear equation. According to Liao, (2004) [37], the Homotopy function is constructed as:

$$
\begin{aligned}
N\left[\psi(\tau, r), u_{0}(\tau), H(\tau), \hbar r\right]= & (1-r)\left\{L\left[\psi(\tau, r)-u_{0}(\tau)\right]\right. \\
& -r \hbar H(\tau) N[\psi(\tau, r)]\},
\end{aligned}
$$

where $\psi(\tau, r)$ is the primary asymptotic solution, $\hbar$ an auxiliary parameter, $H(\tau) \neq 0$ an auxiliary function, $r$ an embedding parameter, $r \epsilon[0,1], L$ an auxiliary linear operator, and $q_{0}$ is an initial guess of the exact solution $q$. The convergence condition highly depends on ones choices of initial guess, auxiliary linear operator, the nonzero auxiliary parameter and the auxiliary function.

The zero order deformation equation (Homotopy equation) is defined when the Homotopy function is set to zero, which leads to a family of equations, a general form of the original nonlinear equation.

$$
(1-r)\left\{L\left[\psi(\tau, r)-q_{0}(\tau)\right]\right\}=r \hbar H(\tau) N[\psi(\tau, r)] .
$$

Now, setting $r=0$, and $r=1$, the zero-order equation reduces to Eqs. (36) and (37), respectively:

$$
\begin{aligned}
& L\left[\psi(\tau, 0)-q_{0}(\tau)\right]=0 \rightarrow \psi(\tau, 0)=q_{0}(\tau), \\
& N[\psi(\tau, 1)]=0 \rightarrow \psi(\tau, 1)=q(\tau) .
\end{aligned}
$$

It is obvious that by varying the embedding parameter from 0 to 1 , the primary solution approaches from the initial guess to the exact solution, $q$. Thus, the following form can be considered for the $m$ th order derivative of $q$ :

$$
q_{m}(\tau)=\left.\sum_{m=1}^{\infty} \frac{1}{m !} \frac{\partial^{m} \psi(\tau, r)}{\partial r^{m}}\right|_{r=0}
$$

Employing Taylor's series, the expanded forms of $\phi(\tau, r)$ and $\omega(r)$ could be written as:

$$
\begin{aligned}
& \psi(\tau, r)=\psi(\tau, 0)+\left.\sum_{m=1}^{\infty} \frac{1}{m !} \frac{\partial^{m} \psi(\tau, r)}{\partial r^{m}}\right|_{r=0}, \\
& r^{m}=\psi(\tau, 0)+\sum_{m=1}^{\infty} q_{m}(\tau) r^{m}, \\
& \omega(r)=\omega_{0}+\left.\sum_{m=1}^{\infty} \frac{1}{m !} \frac{\partial^{m} \omega(r)}{\partial r^{m}}\right|_{r=0}, \\
& r^{m}=\omega_{0}+\sum_{m=1}^{\infty} \omega_{m} r^{m} .
\end{aligned}
$$

With $q_{m}(0)=0$ and $\dot{q}_{m}(0)=0$. In light of Eqs. (37) and (39), one has: 


$$
q(\tau)=q_{0}(\tau)+\sum_{m=1}^{\infty} q_{m}(\tau) .
$$

Now, in order to estimate $q_{m}(\tau)$, one must identify the higher order deformation equation. To do so, Eq. (35) is differentiated with respect to $r$ and the setting $r=0$. By dividing the whole term by $m$ !, the higher order deformation equation for $q$ is structured in the form:

$$
\begin{gathered}
L\left[q_{m}(\tau)\right]=Y_{m} L\left[q_{m-1}(\tau)\right]+\hbar H(\tau) \\
R_{m}\left[q_{1}, \ldots, q_{m-1}, \omega_{0}, \ldots, \omega_{m-1}\right], \\
Y_{m}= \begin{cases}0 & m \leq 1 \\
1 & m>1\end{cases}
\end{gathered}
$$

where:

$$
\begin{aligned}
& R_{m}\left[q_{1}, \ldots, q_{m-1}, \omega_{0}, \ldots, \omega_{m-1}\right] \\
& \quad=\left.\frac{1}{(m-1) !} \frac{\partial^{m-1} N[\psi(\tau, r), \omega(r)]}{\partial r^{m-1}}\right|_{r=0} .
\end{aligned}
$$

Since the right hand side of Eq. (41) is only dependent on $\left\{q_{1}(\tau), q_{2}(\tau), \ldots, q_{m-1}(\tau)\right\}$, the $m$ th-order approximation of $q(\tau)$ can be represented by:

$$
q(\tau) \approx \sum_{i=0}^{m} q_{i}(\tau) .
$$

The corresponding $[m, s]$ pade-approximate about the embedding parameter $r$, for the series of Eq. (39) was proposed by Liao (2004) [37] as a technique to accelerate the convergence of a solution series. Thus, the series of Eq. (39) can be expressed as:

$$
\begin{aligned}
\psi(\tau, r) & =\psi(\tau, 0)+\sum_{m=1}^{\infty} q_{m}(\tau) r^{m} \\
& =\frac{\sum_{k=0}^{m} A_{m, k}(\tau) r^{k}}{\sum_{k=0}^{n} B_{s, k}(\tau) r^{k}},
\end{aligned}
$$

in which, $A_{m, k}$ and $B_{s, k}$ are computed by the following set of approximations:

$$
\left\{q_{0}(\tau), q_{1}(\tau), q_{2}(\tau), \ldots, q_{m+s}(\tau)\right\} .
$$

Now, if $r=1$, the $[m, s]$ Homotopy pade approximant is constructed as:

$$
\phi(\tau, 1)=\frac{\sum_{k=0}^{m} A_{m, k}(\tau)}{\sum_{k=0}^{n} B_{s, k}(\tau)} .
$$

\subsection{Application of homotopy Padé method to the problem}

According to Eqs. (25) and (26), eliminating the external forces, one has:

$$
\begin{aligned}
& N_{y}=\omega_{y}^{2} \frac{\partial^{2} q_{y}}{\partial \tau_{y}^{2}}+\zeta_{y} q_{y}+\beta_{y} q_{y}\left(q_{y}^{2}+\kappa q_{z}^{2}\right), \\
& N_{z}=\omega_{z}^{2} \frac{\partial^{2} q_{z}}{\partial \tau_{z}^{2}}+\zeta_{z} q_{z}+\beta_{z} q_{z}\left(q_{z}^{2} / \kappa+q_{y}^{2}\right), \\
& L_{y}\left[q\left(\tau_{y}, r\right)\right]=\omega_{y 0}^{2}\left(\frac{\partial^{2} q_{y}\left(\tau_{y}, r\right)}{\partial \tau_{y}^{2}}+q_{y}\left(\tau_{y}, r\right)\right), \\
& L_{z}\left[q\left(\tau_{z}, r\right)\right]=\omega_{z 0}^{2}\left(\frac{\partial^{2} q_{z}\left(\tau_{z}, r\right)}{\partial \tau_{z}^{2}}+q_{z}\left(\tau_{z}, r\right)\right),
\end{aligned}
$$

in which $\tau_{y}=\omega_{y} t$, and $\tau_{z}=\omega_{z} t$. By considering:

$$
\begin{aligned}
& u_{y, 0}=a_{y} \cdot \cos \left(\tau_{y}\right), \\
& u_{z, 0}=a_{z} \cdot \cos \left(\tau_{z}\right),
\end{aligned}
$$

where $a_{y}$ and $a_{z}$ are vibration amplitude in $y$ and $z$ directions, respectively, According to Eq. (43), with $H(\tau)=1, \hbar=-1$ and for $m=1$, one may write:

$$
\begin{aligned}
R_{y, m} & =R_{y, 1}=\left(-\omega_{y,(m-1)}^{2} a_{y}+\zeta_{y} a_{y}+\frac{3}{4} \beta_{y} a_{y}^{3}\right. \\
& \left.+\frac{1}{2} \kappa \beta_{y} a_{y} a_{z}^{2}\right) \cos \left(\tau_{y}\right)+\frac{1}{4} \beta_{y} a_{y}^{3} \cos \left(3 \tau_{y}\right) \\
& +\frac{1}{4} \beta_{y} \kappa a_{y} a_{z}^{2} \cos \left(\tau_{y}-2 \tau_{z}\right) \\
& +\frac{1}{4} \beta_{y} k a_{z}^{2} a_{y} \cos \left(\tau_{y}+2 \tau_{z}\right), \\
R_{z, m} & =R_{z, 1}= \\
& \left(-\omega_{z,(m-1)}^{2} a_{z}+\zeta_{z} a_{z}+\frac{3}{4} \beta_{z} a_{z}^{3} / \kappa+\frac{1}{2} a_{z} \beta_{z} a_{y}^{2}\right) \\
& \cos \left(\tau_{z}\right)+\frac{1}{4} \beta_{z} a_{z}^{3} / \kappa \cos \left(3 \tau_{z}\right) \\
& +\frac{1}{4} \beta_{z} a_{z} a_{y}^{2} \cos \left(2 \tau_{y}-\tau_{z}\right) \\
& +\frac{1}{4} \beta_{z} a_{y}^{2} a_{z} \cos \left(2 \tau_{y}+\tau_{z}\right) .
\end{aligned}
$$

To avoid secular terms in the final solution, the sum of the coefficients of $\cos (\tau)$ are taken as equal to zero. In the case of $\kappa=1$, it is a close approximation to consider $\tau_{y}=\tau_{z}=\tau$, so one has:

$$
\begin{aligned}
\omega_{y,(m-1)} & =\omega_{y, 0} \\
& =\sqrt{\zeta_{y}+\frac{3}{4} \beta_{y} a_{y}^{2}+\frac{3}{4} \beta_{y} a_{z}^{2}}, \\
\omega_{z,(m-1)} & =\omega_{z, 0}=\sqrt{\zeta_{z}+\frac{3}{4} \beta_{z} a_{y}^{2}+\frac{3}{4} \beta_{z} a_{z}^{2}} .
\end{aligned}
$$

Solving Eq. (42) for $m=1, q_{y, 1}$ and $q_{z, 1}$ : 
$q_{y, m}=q_{y, 1}=$

$\frac{\left(\left(\beta_{y} a_{y}^{3}+\beta_{y} a_{z}^{2} a_{y}\right) \cos (3 \tau)-\left(\beta_{y} a_{y}^{3}+\beta_{y} a_{z}^{2} a_{y}\right) \cos (\tau)\right)}{24 \beta_{y} a_{y}^{2}+24 \beta_{y} a_{z}^{2}+32 \zeta_{y}}$

$q_{z, m}=q_{z, 1}=$

$$
\frac{\left(\left(\beta_{z} a_{z}^{3}+\beta_{z} a_{y}^{2} a_{z}\right) \cos (3 \tau)-\left(\beta_{z} a_{z}^{3}+\beta_{z} a_{y}^{2} a_{z}\right) \cos (\tau)\right)}{24 \beta_{z} a_{z}^{2}+24 \beta_{z} a_{y}^{2}+32 \zeta_{z}} .
$$

Subsequently, for $m=2$ and calculating the expressions for $R_{y, 2}$ and $R_{z, 2}$, one arrives at results for $\omega_{y, 2}$ and $\omega_{z, 2}$. This procedure is continued to a higher order level for $m=5$, by which an appropriate approximation consistent with numerical data for the natural frequencies of the micro-bridge will be achieved. According to [37], the $[1,1]$ and $[2,2]$ Homotopy-pade approximations for $\omega_{v / w}$ and $q_{v / w}$ are found as:

$$
\omega_{y / z,[1,1]}=\frac{\omega_{y / z, 0}\left(\omega_{y / z, 1}-\omega_{y / z, 2}\right)+\omega_{y / z, 1}^{2}}{\omega_{y / z, 1}-\omega_{y / z, 2}},
$$

Eq. (59) is shown in Box I.

$$
q_{y / z,[1,1]}=\frac{q_{y / z, 0}\left(q_{y / z, 1}-q_{y / z, 2}\right)+q_{y / z, 1}^{2}}{q_{y / z, 1}-q_{y / z, 2}} .
$$

Eq. (61) is shown in Box II.

\section{Numerical results}

In this section, numerical and analytical results are provided in order to investigate the effects of various material and geometrical properties on the static and dynamic responses of a doubly clamped FG tapered micro-beam. The obtained results are compared with the corresponding results calculated by the classical beam theory to clarify the distinctions that have been made in utilizing the length scale parameter. The micro-beam is composed of aluminum (AL) and siliconcarbide ( $\mathrm{SiC})$. The material properties of aluminum are $E_{1}=70 \mathrm{GPa}, v_{1}=0.3$, and $\rho_{1}=2702 \mathrm{~kg} / \mathrm{m}^{3}$, and those of silicon carbide are $E_{0}=427 \mathrm{GPa}, v_{0}=$ 0.17 and $\rho_{0}=3100 \mathrm{~kg} / \mathrm{m}^{3}$ [38]. Since each of the participating constituent materials has a specific value of length scale parameter, it is concluded that the material length scale parameter of the micro-bridge varies along the beam axis. In the present work, the material length scale parameters of silicon-carbide are, respectively, assumed as $l_{1}=10 \mu \mathrm{m}$ and $l_{0}=5 \mu \mathrm{m}$. According to Eq. (19), the effective material length scale parameter can be estimated.

The dimensionless static deflections in the $\hat{z}$ direction for Nonlinear and linear micro-bridges are depicted in Figure 3. It is clear that adding the distributed force in the $y$ direction reduces the nonlinear static deflection of the micro-bridge in the $z$ direction. Further-

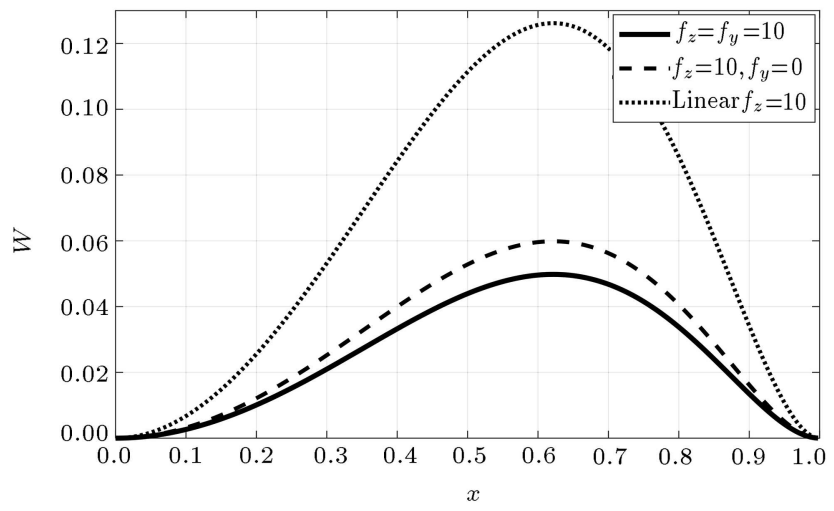

Figure 3. Static deflection along the $x$ axis. FG double tapered micro-bridge, $\varepsilon_{0}=0.04, L / h=20, h / l=3, h=b$, $n=2$.

$$
\omega_{y / z,[2,2]}=\frac{\left[\begin{array}{l}
\omega_{y / z, 0}\left(\omega_{y / z, 2}\left(\omega_{y / z, 3}+\omega_{y / z, 4}\right)+\omega_{y / z, 1}\left(\omega_{y / z, 3}-\omega_{y / z, 4}\right)\right) \\
+\omega_{y / z, 1} \omega_{y / z, 2}\left(2 \omega_{y / z, 3}-\omega_{y / z, 2}\right)+\left(\omega_{y / z, 3}-\omega_{y / z, 4}\right) \omega_{y / z, 1}^{2} \\
-\omega_{y / z, 2}^{3}-\omega_{y / z, 0}\left(\omega_{y / z, 3}^{2}+\omega_{y / z, 2}^{2}\right)
\end{array}\right]}{\omega_{y / z, 4} \omega_{y / z, 2}-\omega_{y / z, 3}^{2}-\omega_{y / z, 4} \omega_{y / z, 1}+\omega_{y / z, 2} \omega_{y / z, 3}+\omega_{y / z, 1} \omega_{y / z, 3}-\omega_{y / z, 2}^{2}} .
$$

Box I

$$
q_{y / z,[2,2]}=\frac{\left[\begin{array}{l}
q_{y / z, 0}\left(q_{y / z, 2}\left(q_{y / z, 3}+q_{y / z, 4}\right)+q_{y / z, 1}\left(q_{y / z, 3}-q_{y / z, 4}\right)\right) \\
+q_{y / z, 1} q_{y / z, 2}\left(2 q_{y / z, 3}-q_{y / z, 2}\right)+\left(q_{y / z, 3}-q_{y / z, 4}\right) q_{y / z, 1}^{2} \\
-q_{y / z, 2}^{3}-q_{y / z, 0}\left(q_{y / z, 3}^{2}+q_{y / z, 2}^{2}\right)
\end{array}\right]}{q_{y / z, 4} q_{y / z, 2}-q_{y / z, 3}^{2}-\omega_{y / z, 4} \omega_{y / z, 1}+q_{y / z, 2} q_{y / z, 3}+q_{y / z, 1} q_{y / z, 3}-q_{y / z, 2}^{2}} .
$$


more, considering the nonlinearity will reduce static deflection. This is due to considering the mid-plane stretching that causes a coupling between equations of both lateral displacements.

Figure 4 plots the frequency ratio (the ratio of non-classical FG tapered-micro-beam frequency to the Non-FG one) for doubly clamped boundary conditions, for different values of power index parameter.

The key point understood from Figure 4 is that, by increasing the power index parameter, the sensitivity to initial deflection heightens. Table 2 expresses the nonlinear natural frequencies in the $z$ direction for various tapered and slender ratios. Since the coupling term is independent of the dimensionless parameter $h / b$ (the ratio of thickness, the edge that is parallel to the $z$ axis to the width, the edge that is parallel to the $y$ axis), changing the size of edge $b$ (width), has

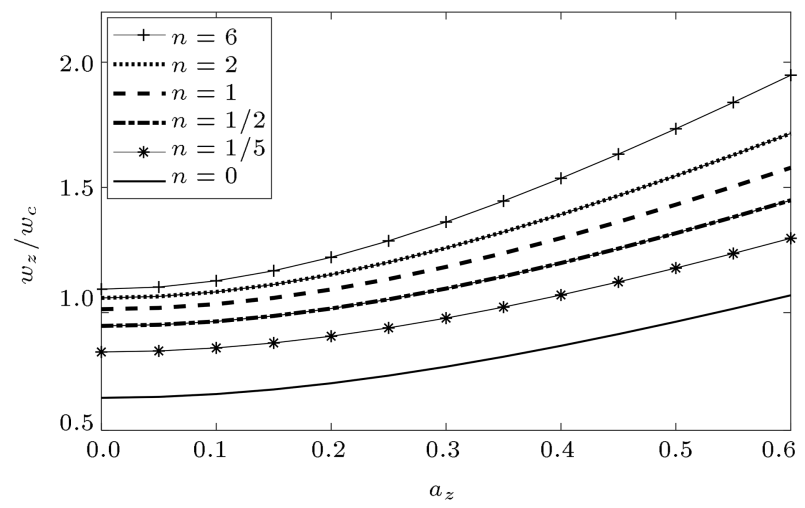

Figure 4. Natural frequency ratio versus initial deflection for different power index parameters. FG micro-bridge, $\omega_{c}=15.119$ a negligible influence on the natural frequency in the $z$ direction. Thus, the effect of this parameter is not reported in these tables. On the other hand, in Table 3, the corresponding values for natural frequencies in the $y$ direction and the effects of changing the size of edge $b$ (changing the $h / b$ parameter) on vibrations along the $y$ axis, can be observed. Numerical results are provided in order to evaluate the accuracy of the analytical HAM method. It should be noted that for a square cross section, the nonlinear natural frequencies are identical for vibrations in both directions, regardless of the given amplitude.

Figure 5 plots the natural frequency ratio (the ratio of a non-classical FG tapered-micro-bridge frequency to the classical one), versus the initial deflection for different values of $h / l$. It is realized that considering

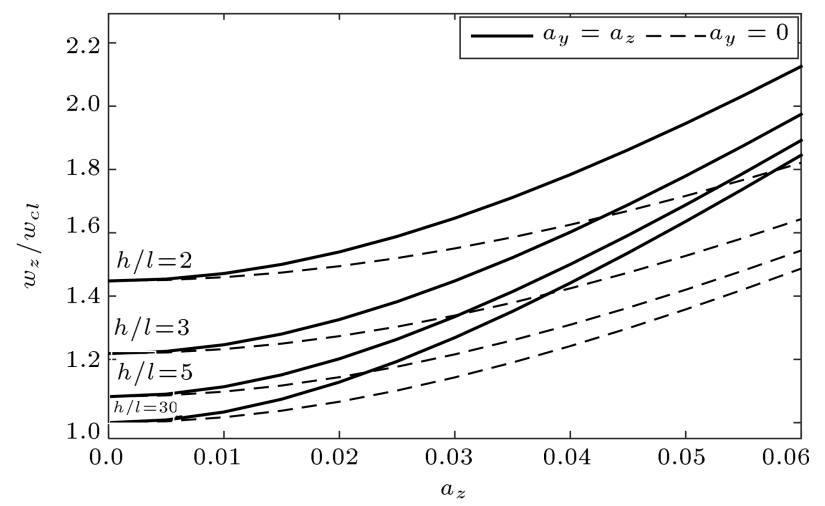

Figure 5. Natural frequency ratio versus initial deflection for different ratios of $h / l$. FG micro-bridge, $\omega_{c l}=13.199$, $\varepsilon_{0}=0.04, L / h=20, h / b=1, n=2$ and $\omega_{c l}$ is the classical natural frequency.

Table 2. Nonlinear natural frequency $\left(\omega_{z} \times \sqrt{\rho_{0} A_{0} L^{4} / E_{0} I_{o y}}\right)$ for a FG micro-bridge and comparison between the present research and numerical Fast Fourier Transform (FFT) results. Relative differences are concluded for different values of $L / h$ and $\epsilon_{0}$. Here we have $n=2, h / l=3$, and $a_{y}=a_{z}=0.03$.

\begin{tabular}{|c|c|c|c|c|c|c|c|c|}
\hline & \multicolumn{2}{|c|}{$L / h=10$} & \multicolumn{2}{|c|}{$L / h=15$} & \multicolumn{2}{|c|}{$L / h=20$} & \multicolumn{2}{|c|}{$L / h=25$} \\
\hline \multirow[t]{2}{*}{$\epsilon_{0}$} & HAM & FFT & HAM & FFT & HAM & FFT & HAM & FFT \\
\hline & \multicolumn{2}{|c|}{ Relative error \% } & \multicolumn{2}{|c|}{ Relative error \% } & \multicolumn{2}{|c|}{ Relative error \% } & \multicolumn{2}{|c|}{ Relative error \% } \\
\hline \multirow[t]{2}{*}{0.2} & 16.139 & 16.297 & 17.266 & 17.396 & 18.720 & 18.868 & 20.427 & 20.647 \\
\hline & \multicolumn{2}{|c|}{0.971} & \multicolumn{2}{|c|}{0.749} & \multicolumn{2}{|c|}{0.783} & \multicolumn{2}{|c|}{1.068} \\
\hline
\end{tabular}

\begin{tabular}{|c|c|c|c|c|c|c|c|c|}
\hline \multirow[t]{2}{*}{0.4} & 16.897 & 16.886 & 17.856 & 17.945 & 19.111 & 19.262 & 20.604 & 20.919 \\
\hline & \multicolumn{2}{|c|}{0.064} & \multicolumn{2}{|c|}{0.496} & \multicolumn{2}{|c|}{0.786} & \multicolumn{2}{|c|}{1.507} \\
\hline \multirow[t]{2}{*}{0.6} & 18.348 & 18.358 & 19.250 & $19.323^{*}$ & 20.439 & 20.556 & 21.865 & 21.970 \\
\hline & \multicolumn{2}{|c|}{0.057} & \multicolumn{2}{|c|}{0.380} & \multicolumn{2}{|c|}{0.573} & \multicolumn{2}{|c|}{0.480} \\
\hline \multirow[t]{2}{*}{0.8} & 19.929 & 19.831 & 20.798 & $20.859^{*}$ & 23.340 & 23.561 & 23.344 & 23.422 \\
\hline & \multicolumn{2}{|c|}{0.493} & \multicolumn{2}{|c|}{0.294} & \multicolumn{2}{|c|}{0.942} & \multicolumn{2}{|c|}{0.336} \\
\hline
\end{tabular}


Table 3. Nonlinear natural frequency $\left(\omega_{y} \times \sqrt{\rho_{0} A_{0} L^{4} / E_{0} I_{o z}}\right)$ for a FG micro-bridge and comparison between the present research and numerical Fast Fourier Transform (FFT) results. Relative differences are concluded for different values of $L / h, h / b$, and $\epsilon_{0}$. Here we have $n=2$ and $h / l=3$.

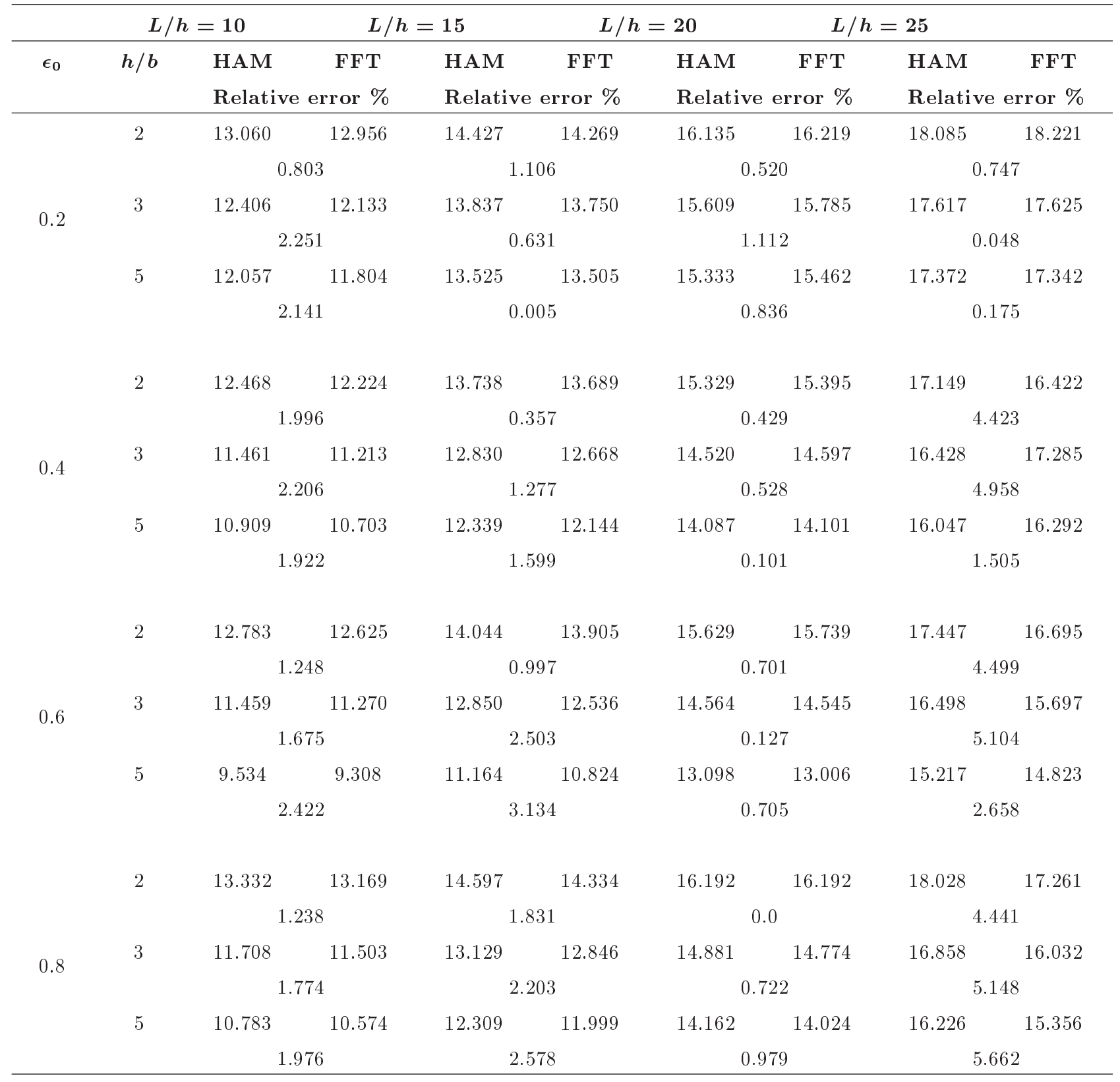

both lateral deflections in calculations, the nonlinear natural frequency has significant effects on dynamic responses.

Due to mid plane stretching, accounting for the third dimension in the vibration problem increases the corresponding natural frequencies. The values obtained for 2D analysis also increase as the initial deflection grows. Furthermore, it is illustrated in Figure 5 that the natural frequency computed by the proposed model here is much higher than that predicted by the classical beam theory. Hence, for larger values of $h / l$ (the ratio of thickness to the length scale parameter of the beam), as shown in Figure 5, the nonlinear responses approach the responses of the classical beam theory.

Table 4 reflects the natural frequency of a micro bridge for a 3D analysis by the MTS method, which is provided by Mojahedi and Rahaeifard (2016) [32] The corresponding results found by the HAM, as well as the numerical solutions, are reported to validate the proposed HAM method in comparison with the MTS 3rd order method. As one may observe, for $a_{y}>0.03$, deviation of the Harmonic Balance method results from the numerical ones increases significantly, while the 
Table 4. Comparison between the present research Homotopy Analysis Method (HAM) and Multiple Time Scale (MTS) method and numerical Fast Fourier Transform (FFT) results in calculating the nonlinear natural frequency $\left(\omega_{z} \times \sqrt{\rho A L^{4} / E I}\right)$ corresponding to a $3 \mathrm{D}$ analysis. Relative differences are concluded for each case. In this calculations we have: $\varepsilon_{0}=1, L / h=30, n=0, h / l=3, \mu=61 \mathrm{GPa}, E=150 \mathrm{GPa}, \rho=2300 \mathrm{~kg} / \mathrm{m}^{3}, v=0.25, h=b$.

\begin{tabular}{lcccccc}
\hline $\boldsymbol{a}_{\boldsymbol{y}}=\boldsymbol{a}_{\boldsymbol{z}}$ & 0.01 & 0.02 & 0.03 & 0.04 & 0.05 & 0.06 \\
FFT & 29.919 & 35.620 & 43.001 & 51.821 & 61.312 & 70.630 \\
\hline HAM & 29.897 & 35.509 & 42.584 & 51.519 & 61.113 & 70.914 \\
HAM relative error \% & $0.073 \%$ & $0.312 \%$ & $0.970 \%$ & $0.583 \%$ & $0.325 \%$ & $0.402 \%$ \\
\hline MTS & 28.658 & 31.283 & 35.656 & 41.779 & 49.652 & 59.273 \\
MTS Relative error\% & $4.215 \%$ & $12.176 \%$ & $17.081 \%$ & $19.378 \%$ & $19.017 \%$ & $25.990 \%$ \\
\hline
\end{tabular}

corresponding error associated with HAM results are negligible. As indicated in Section 1, the results in Table 4 reveal the higher capability of HAM in dealing with large parameter strong nonlinearities and higher vibration amplitude than MTS.

\section{Summary and conclusion}

A nonlinear model for doubly clamped tapered FG micro-beams based on modified couple stress, considering the effects of two coupled bending deformations in lateral directions and also mid-plane stretching, was considered here. After constructing the Lagrangian of the motion, applying the Hamilton principle, the nonlinear 3D equations of motion were derived. In this method, a uni-modal approach was employed to discretize the governing equations and the resultant uni-modal differential equations of motion were solved for static and dynamic responses. The deflections of the single and doubly clamped tapered FG microbeams under different loading conditions were numerically calculated. The corresponding results showed that considering the lateral loading in one direction reduces the static deflection in the other direction. In the case of dynamic analysis, the nonlinear natural frequencies for various geometrical and material compositional properties were calculated using the analytical Homotopy-pade method. It can be observed that by increasing the amplitude of the vibration, the difference between linear and nonlinear natural frequencies increases, and for higher values of $h / l$, both values approach those found through the classical beam theory. Furthermore, it was observed that increasing the power index parameter resulted in higher natural frequency values. The advantages of the Homotopy method in terms of computational cost and accuracy over a classic analytical method, the MTS method (recently employed in the literature to investigate the $3 \mathrm{D}$ vibration of micro beams), in problems with strong nonlinearity and high amplitudes are discussed by a comparison study, which showed the accuracy of the Homotopy Analysis Method (HAM) technique. The corresponding non-linear natural frequencies calculated by FFT as a numerical method were reported to certify the proposed HAM method.

\section{References}

1. Lotfi, M. and Moghimi, M. "Transient behavior and dynamic pull-in instability of electrostaticallyactuated fluid-conveying microbeams", International Journal of Microsyst Technologies, 23(13), pp. 60156023 (2017).

2. Chong, A.C.M., Yang, F., Lam, D.C.C., and Tong, P. "Torsion and bending of micron-scaled structures", Journal of Materials Research, 16(4), pp. 1052-1058 (2001).

3. Stölken, J.S. and Evans, A.G. "A microbend test method for measuring the plasticity length scale", Acta Material, 46(14), pp. 5109-5115 (1998).

4. Ma, Q. and Clarke, D.R. "Size dependent hardness of silver single crystals", J. Mater. Res., 10(4.4), pp. 853-863 (1995).

5. Chong Arthur, C.M. and Lam, D.C.C. "Strain gradient plasticity effect in indentation hardness of polymers", Materials Research, 14(10), pp. 4103-4110 (1999).

6. Yang, F., Chong, A.C.M., Lam, D.C.C., and Tong, P. "Couple stress based strain gradient theory for elasticity", International Journal of Solids and Structures, 39(10), pp. 2731-2743 (2002).

7. Mindlin, R.D. "Second gradient of strain and surfacetension in linear elasticity", International Journal of Solids and Structures, 1(4), pp. 417-438 (1965).

8. Fleck, N.A. and Hutchinson, J.W. "A phenomenological theory for strain gradient effects in plasticity", Journal of the Mechanics and Physics of Solids, 41(12), pp. 1825-1857 (1993).

9. Fleck, N.A. and Hutchinson, J.W. "Strain gradient plasticity", Advances in Applied Mechanics, 33, pp. 295-361 (1997).

10. Fleck, N.A. and Hutchinson, J.W. "A reformulation of strain gradient plasticity", Journal of the Mechanics and Physics of Solids, 49(10), pp. 2245-2271 (2001). 
11. Ansari, R., Faraji Oskouie, M., and Rouhi, H. "Studying linear and nonlinear vibrations of fractional viscoelastic Timoshenko micro-/ nano-beams using the strain gradient theory", Nonlinear Dynamics, 87(1), pp. 695-711 (2016).

12. Li, X., Li, L., Hu, Y., Ding, Z., and Deng, W. "Bending, buckling and vibration of axially functionally graded beams based on nonlocal strain gradient theory", Composite Structures, 165, pp. 250-265 (2017).

13. Mindlin, R.D. and Tiersten, H.F. "Effects of couplestresses in linear elasticity", Archive for Rational Mechanics and Analysis, 11(1), pp. 415-448 (1962).

14. Jafari-talookolaei, R., Ebrahimzade, N., and Rashidijuybari, S. "Bending and vibration analysis of delaminated Bernoulli-Euler microbeams using the modi ed couple stress", Scientia Iranica, 25, pp. 675-688 (2018).

15. Jalali, M.H., Zargar, O., and Baghani, M. "Sizedependent vibration analysis of FG microbeams in thermal environment based on modified couple stress theory", Iranian Journal of Science and Technology, Transactions of Mechanical Engineering, 43, pp. 761771 (2011).

16. Bhattacharya, S. and Das, D. "Free vibration analysis of bidirectional-functionally graded and doubletapered rotating micro-beam in thermal environment using modified couple stress theory", Composite Structures, 215, pp. 471-492 (2019).

17. Baghani, M. "Analytical study on size-dependent static pull-in voltage of microcantilevers using the modified couple stress theory", International Journal of Engineering Science, 54, pp. 99-105 (2012).

18. Lü, C.F., Lim, C.W., and Chen, W.Q. "Size-dependent elastic behavior of FGM ultra-thin films based on generalized refined theory", International Journal of Solids and Structures, 46(5), pp. 1176-1185 (2009).

19. Fu, Y., Du, H., and Zhang, S. "Functionally graded TiN/TiNi shape memory alloy films", Materials Letters, 57(20), pp. 2995-2999 (2003).

20. Rahaeifard, M., Kahrobaiyan, M.H., and Ahmadian, M.T. "Sensitivity analysis of atomic force microscope cantilever made of functionally graded materials", ASME 2009 International Design Engineering Technical Conferences and Computers and Information in Engineering Conference, pp. 539-544 (2009).

21. Bashirpour, M., Forouzmehr, M., and Hosseininejad, S.E. "Improvement of terahertz photoconductive antenna using optical antenna array of $\mathrm{ZnO}$ nanorods", Scientific Reports, pp. 1-8 (2019).

22. Baghani, M. and Fereidoonnezhad, B. "Limit analysis of FGM circular plates subjected to arbitrary rotational symmetric loads using von-Mises yield criterion", Acta Mechanica, 224(8), pp. 1601-1608 (2013).

23. Baghani, M., Mazaheri, H., and Salarieh, H. "Analysis of large amplitude free vibrations of clamped tapered beams on a nonlinear elastic foundation", Applied Mathematical Modelling, 38(3), pp. 1176-1186 (2014).
24. Sadeghi, H., Baghani, M., and Naghdabadi, R. "Strain gradient elasticity solution for functionally graded micro", International Journal of Engineering Science, 50(1), pp. 20-23 (2011).

25. Bashirpour, M., Kefayati, A., Kolahdouz, M., and Aghababa, H. "Tuning the electronic properties of symetrical and asymetrical boron nitride passivated graphene nanoribbons: Density function theory", Journal of Nano Research, 54, pp. 35-41 (2018).

26. Baghani, M., Mazaheri, H., and Salarieh, H. "Analysis of large amplitude free vibrations of clamped tapered beams on a nonlinear elastic foundation", Applied Mathematical Modelling, 38(3), pp. 1176-1186 (2014).

27. Raju, S.S., Umapathy, M., and Uma, G. "High-output piezoelectric energy harvester using tapered beam with cavity", Journal of Intelligent Material Systems and Structures, 29(5), pp. 1-16 (2017).

28. Mohammadsalehi, M., Zargar, M., and Baghani, M. "Study of non-uniform viscoelastic nanoplates vibration based on nonlocal first-order shear deformation theory", Meccanica, 52(4-5), pp. 1063-1077 (2017).

29. Zhao, X.W., Hu, Z.D., and van der Heijden, G.H.M. "Dynamic analysis of a tapered cantilever beam under a travelling mass", Meccanica, 50(6), pp. 1419-1429 (2015).

30. Mojahedi, M., Ahmadian, M.T., and Firoozbaksh, K. "Effects of casimir and van der waals forces on the pull-in instability of the nonlinear micro and nanobridge gyroscopes", International Journal of Structural Stability and Dynamics, 14(2), pp. 135-159 (2014).

31. Mojahedi, M., Ahmadian, M.T., and Firoozbakhsh, K. "The influence of the intermolecular surface forces on the static deflection and pull-in instability of the micro/nano cantilever gyroscopes", Composites Part B: Engineering, 56, pp. 336-343 (2014).

32. Mojahedi, M. and Rahaeifard, M. "A size-dependent model for coupled 3D deformations of nonlinear microbridges", International Journal of Engineering Science, 100, pp. 171-182 (2016).

33. Park, S.K. and Gao, X. "Bernoulli-Euler beam model based on a modified couple stress theory", IOP science, 2355, pp. 1-5 (2006).

34. Liao, S., Homotopy Analysis Method in Nonlinear Differential Equations, Springer (2011).

35. Carrera, E., Giunta, G., and Petrolo, M., Beam Structures, Beam Structures: Classical and Advanced Theories, John Wiley and S ons (2011).

36. Baghani, M., Mohammadsalehi, M., and Dabaghian, P.H. "Analytical couple-stress solution for sizedependent large-amplitude vibrations of FG taperednanobeams", Latin American Journal of Solids and Structures, 13(1), pp. 95-118 (2014).

37. Liao, S., Beyond Perturbation: An Introduction to the Homotopy Analysis Method, Chapman \& Hall/CRC (2004). 
38. Zhang, B., He, Y., Liu, D., Gan, Z., and Shen, L. "Sizedependent functionally graded beam model based on an improved third-order shear deformation theory", European Journal of Mechanics, A/Solids, 47, pp. 211-230 (2014).

\section{Biographies}

Shadi Haddad obtained her BS degree in Mechanical Engineering from Shahid Chamran University, Ahvaz, Iran, in 2015, and her MS degree in Mechanical Engineering from the Department of Mechanical Engineering at Tehran University, Tehran, Iran, in 2018. Her research interests include vibration analysis, system dynamics and controls, fault detection, microelectromechanical systems.

Mostafa Baghani received his BS degree in Mechanical Engineering from the University of Tehran,
Iran, in 2006, and MS and PhD degrees in Mechanical Engineering from the Department of Mechanical Engineering at Sharif University of Technology, Tehran, Iran, in 2008 and 2012, respectively. He is now Assistant Professor in the School of Mechanical Engineering at the University of Tehran. His research interests include solid mechanics, nonlinear finite element method, and shape-memory materials constitutive modeling.

Mohammad Reza Zakerzadeh received his BS, MS, and PhD degrees in Mechanical Engineering from Sharif University of Technology, Tehran, Iran, in 2002, 2005 and 2012, respectively. Since 2012, he has been with the School of Mechanical Engineering at the College of Engineering at the University of Tehran. He is now Associate Professor and his research activities include smart material and structures, shape memory alloy (SMA), dynamics, control and robotics. 